Urol. Prax. 2020 $22: 142-146$

https://doi.org/10.1007/s41973-020-00116-9

Online publiziert: 9 . November 2020

(c) Der/die Autor(en) 2020

Andreas Katsios - George N. Thalmann - Tobias Gross

Urologische Universitätsklinik, Inselspital, Bern, Schweiz

\title{
Die unklare Nierenraumforderung: wie weiter?
}

werden Nierenraumforderungen, wie bereits erwähnt, als solid oder zystisch klassifiziert. Ein Ziel der Bildgebung renaler Tumoren ist die möglichst präzise Voraussage, ob es sich bei der gefundenen Läsion um einen benignen oder malignen Prozess handelt, welcher evtl. zeitnah einer gezielten Therapie bedarf. Hauptproblem bei der Unterscheidung zwischen gutartigen und bösartigen Nierentumoren in allen bildgebenden Verfahren sind v. a. überlappende Bildcharakteristika in beiden Gruppen [2-4]. Die primäre Abklärung solider Tumoren erfolgt durch kontrastmittelverstärkte Computertomographie des Abdomens und zur Komplettierung des Stagings auch vom Thorax [5]. Da für die Durchführung einer Computertomographie gewisse Limitationen bestehen, wie die Strahlenexposition, Kontrastmittelallergie und Nephrotoxizität, ist die Bildgebung mittels Magnetresonanztomographie (MRT) empfohlen [5]. Insbesondere weist die MRT für die Abklärung von kleinen zystischen Läsionen eine höhere Sensitivität und Spezifität als das CT auf und kann zusätzliche Informationen über die Ausbreitung eines Tumorthrombus der V. cava inferior liefern [5-8]. Jedoch ist die MRT nicht für jeden Patienten anwendbar. Hohe Kosten, relativ lange Untersuchungszeit, nicht MR-taugliche Implantate, Klaustrophobie oder Gadoliniumallergie sind Einschränkungen der Nutzung der MRT.

Eine wertvolle Alternative stellt der kontrastmittelverstärkte Ultraschall (CEUS) dar. Dabei werden als Kontrastmittel gasgefüllte Mikrobläschen i.v. appliziert, die danach über die Lunge abgeatmet werden. Dies hat den grossen
Vorteil, dass CEUS bei Patienten mit Niereninsuffizienz angewendet werden kann. Ausserdem besteht keine Strahlenexposition, anaphylaktische Reaktionen sind sehr selten und die Untersuchungskosten niedrig [9]. Jedoch ist dieses Bildgebungsverfahren untersucherabhängig und somit schwierig reproduzierbar. Einen besonderen Stellenwert bekommt die Untersuchung bei der Beurteilung von komplizierten zystischen Läsionen [10, 11]. Zystische Tumoren werden nach Bosniak klassifiziert [12]. BosniakI- und -II-Zysten gelten als benigne und bedürfen keiner Therapie, Bosniak-IIIund -IV-Zysten sind primär malignitätssuspekt und sollten analog einem soliden Tumor behandelt werden. Eine Sonderkategorie stellen die IIF-Zysten dar, welche weder klar benigne noch maligne sind und deshalb im Verlauf kontrolliert werden sollten ( $\bullet$ Tab. 1).

Auch bei soliden Nierentumoren ist die eindeutige und sichere Differenzierung zwischen gut- und bösartig mittels Ultraschall - trotz Einsatz moderner Techniken wie Farbdoppler und kontrastverstärktem Ultraschall - oftmals nicht ausreichend genau möglich. Mit Ausnahme des „klassischen“, fettreichen Angiomyolipoms bereiten solide Nierentumoren aber auch den anderen bildgebenden Verfahren wie MRT und CT Probleme bei der sicheren Diagnose. Es erstaunt somit nicht, dass in der Literatur bis $\mathrm{zu} 20 \%$ der resezierten soliden RF benigne sind [13]. Aus diesem Grund ist für ältere Patienten mit Komorbiditäten bei kleinen Tumoren $(<4 \mathrm{~cm})$ auch das aktive Überwachen eine mögliche Strategie, insbesondere da sich in dieser Population eine tiefe 


\begin{tabular}{|c|c|c|}
\hline Typ I & $\begin{array}{l}\text { Einfache, flüssigkeitsgefüllte Zyste }\left(<20 \mathrm{HU}^{\mathrm{a}}\right) \\
\text { Gleichmässige Begrenzung, keine sichtbare Wand } \\
\text { Keine Kontrastmittelanreicherung }\end{array}$ & $\begin{array}{l}\text { Einfache Zyste } \\
\text { Benigne }\end{array}$ \\
\hline Typ II & $\begin{array}{l}\text { Feine Septen } \leq 2, \text { keine sichtbare Wand } \\
\text { Feine Kalzifizierungen }(<1 \mathrm{~mm}) \\
\text { Keine Kontrastmittelanreicherung }\end{array}$ & $\begin{array}{l}\text { Komplexe Zyste } \\
\text { Benigne }\end{array}$ \\
\hline Typ IIF & $\begin{array}{l}\text { Feine Septen }(>3) \text { mit zarter Zystenwand }(\leq 1 \mathrm{~mm}) \\
\text { Dickere Kalzifizierungen }(>1 \mathrm{~mm}) \\
\text { Moderate Kontrastmittelanreicherung } \\
\text { Hyperdense Läsion }>3 \mathrm{~cm} \text { oder intraparenchymatöse Lokalisation }\end{array}$ & $\begin{array}{l}\text { Komplexe, verdächtige Zyste } \\
\text { Malignitätsrisiko } \leq 5 \% \\
\text { Verlaufskontrolle erforderlich (F: Follow-up) }\end{array}$ \\
\hline Typ III & $\begin{array}{l}\text { Zahlreiche und/oder verdickte Septen } \\
\text { Gleichmässig verdickte und diskret irreguläre Zystenwand } \\
\text { Kontrastmittelanreicherung in Zystenwand/Septen }\end{array}$ & $\begin{array}{l}\text { Zystischer Tumor } \\
\text { Malignitätsrisiko >50\% } \\
\text { Chirurgische Intervention oder „active surveillance” }\end{array}$ \\
\hline Typ IV & $\begin{array}{l}\text { Verdickte und sehr irreguläre Zystenwand } \\
\text { Papilläre oder noduläre Wandformationen } \\
\text { Kontrastmittelanreicherung in den soliden Gewebeanteilen }\end{array}$ & $\begin{array}{l}\text { Zystisches Karzinom } \\
\text { Malignitätsrisiko } 100 \% \\
\text { Chirurgische Intervention erforderlich }\end{array}$ \\
\hline
\end{tabular}

karzinomspezifische Mortalität gezeigt hat [14].

\section{Biopsie}

Die Rolle der Biopsie in der Diagnostik von renalen Raumforderungen wird kontrovers diskutiert. Die Biopsie kann bei radiologisch unklaren, soliden Tumoren wichtige Informationen liefern. Jedoch bleibt auch nach Biopsie eine gewisse diagnostische Unsicherheit, weshalb in Zeiten der minimal-invasiven Nierenteilresektion zunehmend auf die Biopsieentnahme verzichtet wird. Eine Indikation besteht jedoch vor ablativen Verfahren sowie bei der Auswahl eines bestmöglichen onkologischen und chirurgischen Therapieregimes beim metastasierten Stadium $[15,16]$. Gemäss den aktuellen Richtlinien sollte eine Biopsie in Lokalanästhesie ultraschall- oder CT-gesteuert im koaxialen Verfahren durchgeführt werden. Das Risiko der Tumorzellverbreitung bei diesem Verfahren ist selten und von unklarer Signifikanz [17]. Zystische Raumforderungen unklarer Dignität sollten aufgrund der erhöhten Rate eines „sampling error“ nicht biopsiert werden $[18,19]$.

\section{Maligne Nierentumoren}

\section{Nierenzellkarzinom}

Das Nierenzellkarzinom (• Abb. 1) macht je nach Geschlecht ca. 3\% aller malignen Erkrankungen aus. In Europa, Nordamerika und Australien sind die Inzidenzraten höher als in Asien und Afrika. In Europa sind Männer mit einer Inzidenz von ca. 26/100.000 deutlich häufiger als Frauen mit einer Inzidenz von ca. 12/100.000 betroffen. Das mittlere Erkrankungsalter liegt zwischen 60 und 70 Jahren [20]. Als erworbene Risikofaktoren für die Entstehung eines Nierenzellkarzinoms werden hauptsächlich Rauchen, Übergewicht und arterielle Hypertonie beschrieben [21]. In rund 5-8\% der Patienten tritt das Nierenzellkarzinom im Rahmen einer hereditären Störung auf. Die bekanntesten assoziierten Syndrome sind das Von-HippelLindau-Syndrom, das Birt-Hogg-DubéSyndrom, tuberöse Sklerose und hereditäres nichtpolypöses kolorektales Karzinom.

Das Nierenzellkarzinom macht etwa $90 \%$ der malignen Nierentumoren aus und ist eine heterogene Erkrankung. Histologisch dominieren das klarzellige (ca. 80-90\%), das papilläre (Typ I und II, ca. 6-15\%) und das chromophobe (ca. 2-5\%) Karzinom [4].

Die Prognose in allen diesen Subtypen verschlechtert sich bei höherem TNM-Stadium und WHO/ISUP(World Health Organisation/International Society of Urological Pathology)- oder Fuhrman Grad. Die 5-Jahres-Gesamtüberlebensrate für alle Subtypen beträgt $49 \%$.
Die wirksamsten kausalen Therapieverfahren sind die Operation und die medikamentöse Therapie. Die Operation ist die einzige kurative Option. Das Gesamtbehandlungskonzept soll vor der ersten therapeutischen Maßnahme festgelegt werden. Es wird zwischen den lokal begrenzten, lokal fortgeschrittenen und metastasierten Stadien unterschieden.

Therapie der Wahl beim lokal begrenzten Nierenzellkarzinom ist die chirurgische Resektion. Hier stehen die radikale und die partielle Nephrektomie zur Verfügung. Während die onkologischen Resultate für beide Methoden bei niedrigem $\mathrm{T}$-Stadium vergleichbar sind [22], ist das Risiko für die Entwicklung von kardiovaskulären Erkrankungen nach der Teilnephrektomie niedriger [23, 24]. Weitere Indikationen für eine partielle Nephrektomie sind eine anatomische oder funktionelle Einzelniere, das erhöhte Risiko für eine Niereninsuffizienz aus anderer Ursache (z.B. Hypertonie, Diabetes mellitus), hereditäre Nierenzellkarzinomsyndrome und T1-Stadium. Im Stadium T2 ist der Erfolg einer partiellen Nephrektomie von der sorgfältigen Patientenselektion und der chirurgischen Expertise abhängig.

Sowohl die radikale als auch die partielle Nephrektomie können offen oder minimal-invasiv (retroperitoneoskopisch, laparoskopisch, roboterassistiert) durchgeführt werden. Die minimal-invasive Nephrektomie kann das Risiko perioperativer Morbidität reduzieren 
[23]. Allerdings fehlen große randomisierte Studien zur Gleichwertigkeit der offenen und der laparoskopischen, partiellen Nephrektomie unter onkologischen Gesichtspunkten. Minimalinvasive Nierenteilresektionen sollten an ausgewählten Zentren mit entsprechender Expertise durchgeführt werden. Wenn immer onkologisch vertretbar, sollte dem Nierenerhalt mittels partieller Nephrektomie der Vorzug gegenüber dem radikalen Verfahren gegeben werden. Obwohl die Datenlage bezüglich Erhalt der Nierenfunktion kontrovers ist, zeigte die einzige randomisierte Studie, in welcher die Nierenteilresektion mit der radikalen Nephrektomie bei Tumoren mit einem Durchmesser $\leq 5 \mathrm{~cm}$ verglichen wurde, eine Reduktion der moderaten Niereninsuffizienz bei Patienten, welche einer Nierenteilresektion unterzogen worden waren, interessanterweise war jedoch die Inzidenz von höhergradiger Niereninsuffizienz in beiden Gruppen vergleichbar [24].

Die simultane Adrenalektomie ist nur erforderlich bei bildgebendem oder intraoperativem Verdacht auf Tumorinfiltration oder Metastasen [25]. Der Stellenwert der Lymphknotenresektion ist beim lokal begrenzten Stadium nach wie vor nicht mit abschliessender Sicherheit für alle Tumorstadien geklärt, jedoch zeigte eine randomisierte Studie in dieser Population keinen Einfluss auf die Prognose und wird deshalb nur bei Patienten mit bildgebendem oder intraoperativem Verdacht auf Lymphknotenbeteiligung empfohlen [26].

Therapeutische Ansätze als Alternativen zur chirurgischen Resektion sind verschiedene physikalische Verfahren mit perkutaner, gezielter Therapie unter bildgebender Kontrolle. Die bekanntesten sind die Kryotherapie und die Radiofrequenzablation, wobei die Qualität der verfügbaren Daten keine endgültigen Rückschlüsse auf die Morbidität und die onkologischen Ergebnisse zulässt [5]. Ein anderes lokales Therapieverfahren ist die Embolisierung des Tumors. Sie wird als palliative Maßnahme bei persistierender oder massiver Makrohämaturie eingesetzt, wenn weder eine Operation noch eine systemische Therapie aufgrund des schlechten Allgemeinzu-

Urol. Prax. 2020 - 22:142-146 https://doi.org/10.1007/s41973-020-00116-9

(c) Der/die Autor(en) 2020

\section{A. Katsios · G. N. Thalmann · T. Gross \\ Die unklare Nierenraumforderung: wie weiter?}

\section{Zusammenfassung}

Raumforderungen der Niere sind eine heterogene Gruppe von benignen und malignen Tumoren. Eine entscheidende Rolle bei der weitergehenden Differenzierung und auch Überwachung spielt die Bildgebung. In gewissen Fällen kann eine Biopsie sinnvoll sein, insbesondere bei der Abklärung von metastasierten Leiden oder vor ablativen Verfahren zur Gewinnung einer Histologie. Bei T1/T2-Tumoren sollte, wenn immer vertretbar, eine Nierenteilresektion angestrebt werden, die minimal-invasiven Operationen sind zunehmend die Modalität der Wahl, jedoch sollte die minimal-invasive Methode nicht auf Kosten eines Nierenerhalts forciert werden. Bei älteren und komorbiden Patienten mit kleinen Nierentumoren ist die aktive Überwachung eine valide Alternative zur unmittelbaren Chirurgie.

\section{Schlüsselwörter}

Nierentumor · Nierenzellkarzinom · Onkozytom $\cdot$ Nierenteilresektion $\cdot$ Nephrektomie

\section{Masse rénale de nature indéterminée: quelle est la marche à suivre?}

\section{Résumé}

Les masses rénales sont un groupe hétérogène de tumeurs bénignes ou malignes. Un rôle décisif pour la différenciation ainsi que pour la surveillance par la suite revient à l'imagerie médicale. Une biopsie peut être utile dans certains cas, notamment pour le diagnostic de maladies métastatiques ou pour l'évaluation histologique avant une chirurgie ablative. Une néphrectomie partielle doit toujours être visée lors de tumeurs T1/T2 si elle est justifiable; les opérations mini-invasives sont de plus en plus la modalité préférée, mais ne

stands möglich sind. Zusätzlich kann bei gebrechlichen Patienten mit Komorbiditäten und kleinen Nierentumoren eine aktive Überwachung angeboten werden.

Beilokal fortgeschrittenen Stadien mit venösem Tumorthrombus wird die Exzision des Tumorthrombus empfohlen, nicht aber eine adjuvante Systemtherapie [5].

Beim metastasierten Nierenzellkarzinom steht die medikamentöse Tumortherapie (angiogenesehemmende Multityrosinkinaseinhibitoren [TKI], mTOR-Inhibitoren, Kombination Interferon-alpha und VEGF-Antikörper Bevacizumab sowie aktuell neuere TKI und Checkpointinhibitoren) im Vordergrund. Eine ergänzende, zytoreduktive Nephrektomie kann als Element eines multimodalen Therapiekonzeptes in Abhängigkeit vom Progressionsrisiko im doivent pas être choisies aux dépens d'une conservation du rein. Lors de tumeurs rénales de petites dimensions chez des patients âgés ou présentant des comorbidités, la surveillance active est une alternative valide au traitement chirurgical immédiat.

\section{Mots clés}

Tumeur rénale - Carcinome à cellules rénales . Oncocytome - Résection partielle du rein . Néphrectomie interdisziplinären Tumorboard diskutiert werden. Weitere lokale Therapieverfahren wie Metastasenresektion oder stereotaktische Bestrahlung ossärer oder zerebraler Metastasen können im Rahmen symptomorientierter Maßnahmen eingesetzt werden.

Die Nachsorge nach kurativer Operation eines Nierenzellkarzinoms sollte risikoadaptiert erfolgen, mit dem Ziel der frühzeitigen Diagnose eines Lokalrezidivs, der frühzeitigen Diagnose eines kontralateralen Rezidivs und des Nachweises von Metastasen. Weitere Ziele sind die Überwachung von therapieinduzierten Nebenwirkungen und der Nierenfunktion. 

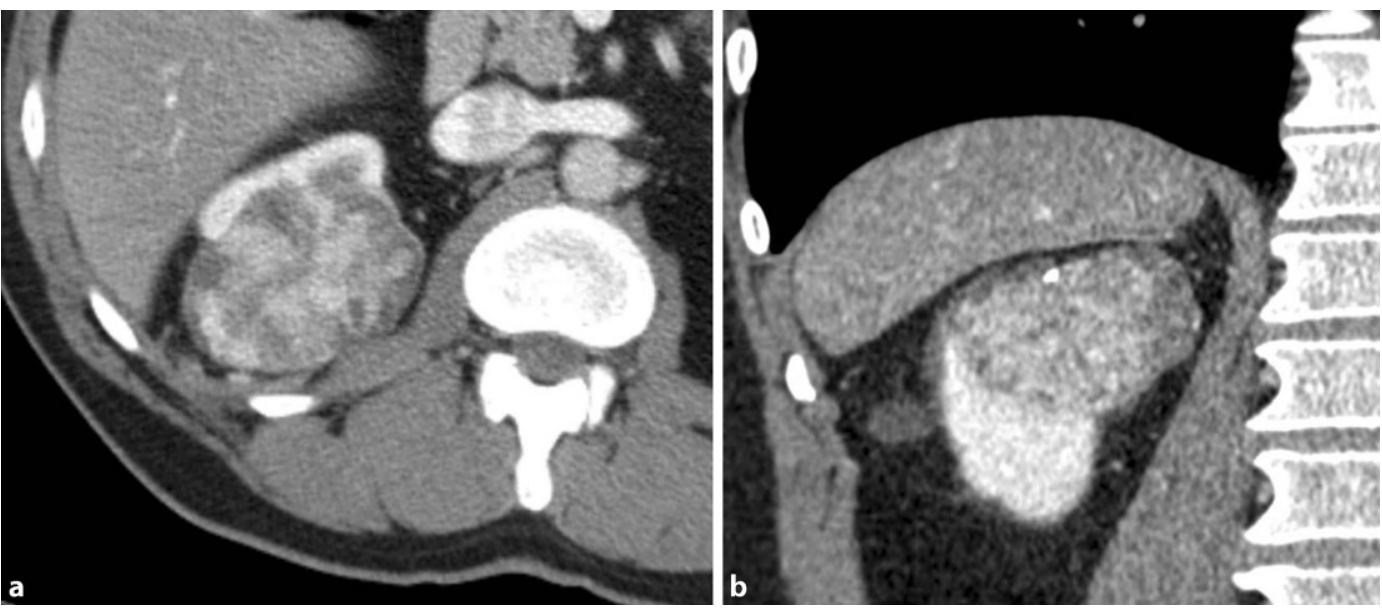

Abb. $1<$ Nierenzellkarzinom
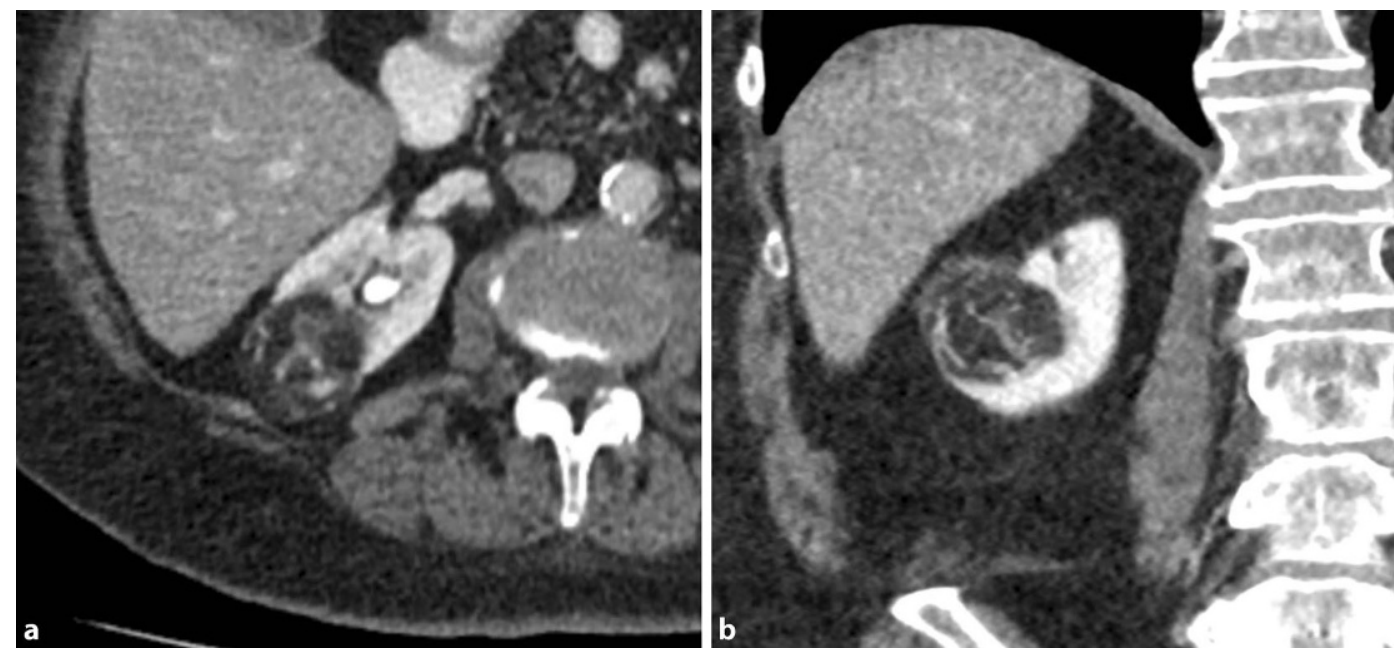

Abb. $2 \triangleleft$ Angiomyolipom. (Mit freundl.Genehmigung von Dr. med. Verena Carola Obmann)

\section{Benigne Nierentumoren}

\section{Angiomyolipom}

Das Angiomyolipom (AML; • Abb. 2) ist ein gutartiger, mesenchymaler Tumor und zu $20 \%$ mit einer tuberösen Sklerose assoziiert. Die Prävalenz beträgt ca. 0,4 \%, und nur $5 \%$ dieser Patienten haben multiple Befunde [27]. Aufgrund des hohen Fettgehalts werden die Läsionen häufig mittels Ultraschall, CT und MRT diagnostiziert. Die fettarmen AML können allerdings nicht mittels Bildgebung zuverlässig diskriminiert werden, sodass eine perkutane Biopsieentnahme die Diagnose liefern kann. Bei Patienten mit tuberöser Sklerose kann eine multizentrische Ausbreitung mit Befall und Vergrösserung von Lymphknoten vorliegen. In seltenen Fällen kann ein nichtmaligner Thrombus in der Nierenvene oder der V. cava inferior gefunden werden.
Das epitheloide Angiomyolipom ist eine sehr seltene Variante und potenziell maligne mit aggressivem Verlauf [28]. In den meisten Fällen wachsen Angiomyolipome langsam und haben eine niedrige Morbidität [29]. Der traditionell verwendete 4-cm-Grenzwert sollte nicht per se zu einer aktiven Behandlung führen. Bei persistierenden Schmerzen, rupturiertem AML mit akuter oder rezidivierender Blutung, sehr grossen Befunden oder Frauen im gebärfähigen Alter sollten eine selektive arterielle Embolisation oder in seltenen Fällen eine Nierenteilresektion durchgeführt werden [29]. Eine Grössenreduktion von bilateralen Befunden bei Patienten mit tuberöser Sklerose kann durch die Anwendung von Everolimus erzielt werden [30].

\section{Onkozytom}

Das Onkozytom repräsentiert etwa 3-7 \% aller Nierentumoren. Die Abgrenzung zum Nierenzellkarzinom kann sowohl histologisch als auch in der Bildgebung herausfordernd sein [31]. Insbesondere bei Patienten mit mehreren onkozytomverdächtigen Läsionen muss beachtet werden, dass in einem substanziellen Anteil der Patienten ein simultanes Nierenzellkarzinom vorliegen kann [32].

\section{Korrespondenzadresse}

\section{Andreas Katsios}

Urologische Universitätsklinik, Inselspital 3010 Bern, Schweiz

andreas.katsios@insel.ch

Funding. Open access funding provided by University of Bern 


\section{Einhaltung ethischer Richtlinien}

Interessenkonflikt. A. Katsios, G.N. Thalmann und T.Gross geben an, dass kein Interessenkonflikt besteht.

Für diesen Beitrag wurden von den Autoren keine Studien an Menschen oder Tieren durchgeführt. Für die aufgeführten Studien gelten die jeweils dort angegebenen ethischen Richtlinien.

Open Access. Dieser Artikel wird unter der Creative Commons Namensnennung 4.0 International Lizenz veröffentlicht, welche die Nutzung, Vervielfältigung, Bearbeitung, Verbreitung und Wiedergabe in jeglichem Medium und Format erlaubt, sofern Sie den/die ursprünglichen Autor(en) und die Quelle ordnungsgemäß nennen, einen Link zur Creative Commons Lizenz beifügen und angeben, ob Änderungen vorgenommen wurden.

Die in diesem Artikel enthaltenen Bilder und sonstige Drittmaterial unterliegen ebenfalls der genannten Creative Commons Lizenz, sofern sich aus der Abbildungslegende nichts anderes ergibt. Sofern das betreffende Material nicht unter der genannten Creative Commons Lizenz steht und die betreffende Handlung nicht nach gesetzlichen Vorschriften erlaubt ist, ist für die oben aufgeführten Weiterverwendungen des Materials die Einwilligung des jeweiligen Rechteinhabers einzuholen.

Weitere Details zur Lizenz entnehmen Sie bitte der Lizenzinformation auf http://creativecommons.org/ licenses/by/4.0/deed.de.

\section{Literatur}

1. Patard JJ, Leray E, Rodriguez A et al (2003) Correlation between symptom graduation, tumor characteristics and survival in renal cell carcinoma. EurUrol 44:226-232

2. Wobker SE, Williamson SR (2017) Modern pathologic diagnosis of renal oncocytoma.J Kidney Cancer VVHL 4:1-12

3. Bhatt NR, Davis NF, Flynn Retal (2015) Dilemmas in diagnosis and natural history of renal oncocytoma and implications for management. Can Urol Assoc」 9:E709-712

4. Moch H, Cubilla AL, Humphrey PA et al (2016) The 2016 WHO classification of tumours of the urinary system and male genital organs-part A: renal, penile, and testicular tumours. Eur Urol 70:93-105

5. Ljungberg B, Albiges L, Abu-Ghanem Yet al (2019) European Association of Urology Guidelines on Renal Cell Carcinoma: The 2019 Update. Eur Urol 75:799-810

6. Janus CL, Mendelson DS (1991) Comparison of MR and CT for study of renal and perirenal masses. Crit Rev Diagn Imaging 32:69-118

7. Krestin GP, Gross-Fengels W, Marincek B (1992) The importance of magnetic resonance tomography in the diagnosis and staging of renal cell carcinoma. Radiologe 32:121-126

8. Mueller-Lisse UG, Mueller-Lisse UL (2010) Imaging of advanced renal cell carcinoma. World J Urol 28:253-261

9. Cokkinos DD, Antypa EG, Skilakaki M et al (2013) Contrast enhanced ultrasound of the kidneys: what is it capable of? Biomed Res Int 2013:595873

10. Defortescu G, Cornu JN, Béjar S et al (2017) Diagnostic performance of contrast-enhanced ultrasonographyandmagneticresonanceimaging for the assessment of complex renal cysts: a prospective study. Int JUrol 24:184-189

11. Clevert DA, Minaifar N, Weckbach S et al (2008) Multislice computed tomography versus contrastenhanced ultrasound in evaluation of complex cystic renal masses using the Bosniak classification system. Clin Hemorheol Microcirc 39:171-178

12. Bosniak MA (1997) The use of the Bosniak classification system for renal cysts and cystic tumors. JUrol 157:1852-1853

13. Lane BR, Babineau D, Kattan MW et al (2007) A preoperative prognostic nomogram for solid enhancing renal tumors $7 \mathrm{~cm}$ or less amenable to partial nephrectomy. JUrol 178:429-434

14. Lane BR, Abouassaly R, Gao T et al (2010) Active treatment of localized renal tumors may not impact overall survival in patients aged 75 years or older.Cancer 116:3119-3126

15. Maturen KE, Nghiem HV, CaoiliEMetal (2007)Renal mass core biopsy: accuracy and impact on clinical management. AJR Am J Roentgenol 188:563-570

16. AbelEJ,CulpSH, Matin SF etal (2010) Percutaneous biopsy of primary tumor in metastatic renal cell carcinoma to predict high risk pathological features: comparison with nephrectomy assessment. JUrol 184:1877-1881

17. Macklin PS, Sullivan ME, Tapping CR et al (2019) Tumour seeding in the tract of percutaneous renal tumour biopsy: a report on seven cases from a UK tertiary referral centre. Eur Urol 75:861-867

18. Richard PO, Jewett MA, Bhatt JR et al (2015) Renal tumor biopsy for small renal masses: a singlecenter 13-year experience. Eur Urol 68:1007-1013

19. Marconi L, Dabestani S, Lam TB et al (2016) Systematic review and meta-analysis of diagnostic accuracy of percutaneous renal tumour biopsy. Eur Urol 69:660-673

20. Ferlay J, Steliarova-Foucher E, Lortet-Tieulent J et al (2013) Cancer incidence and mortality patterns in Europe: estimates for 40 countries in 2012. Eur $J$ Cancer 49:1374-1403

21. Al-Bayati O, Hasan A, Pruthi D et al (2019) Systematic review of modifiable risk factors for kidney cancer. Urol Oncol 37:359-371

22. Van Poppel H, Da Pozzo L, Albrecht W et al (2011) A prospective, randomised EORTC intergroup phase 3 study comparing the oncologic outcome of elective nephron-sparing surgery and radical nephrectomy for low-stage renal cell carcinoma. EurUrol 59:543-552

23. Maclennan S, Imamura M, Lapitan MC et al (2012) Systematic review of perioperative and quality-oflife outcomes following surgical management of localised renal cancer. Eur Urol 62:1097-1117

24. Scosyrev E, Messing EM, Sylvester R et al (2014) Renal function after nephron-sparing surgery versus radical nephrectomy: results from EORTC randomized trial 30904. Eur Urol 65:372-377

25. Lane BR, Tiong HY, Campbell SC et al (2009) Management of the adrenal gland during partial nephrectomy. J Urol 181:2430-2436 (discussion 2436-2437)

26. Blom JH, Van Poppel H, Maréchal JM et al (2009) Radical nephrectomy with and without lymph-node dissection: final results of European Organization for Research and Treatment of Cancer (EORTC) randomized phase 3 trial 30881. Eur Urol 55:28-34

27. Fittschen A, Wendlik I, Oeztuerk S et al (2014) Prevalence of sporadic renal angiomyolipoma: a retrospective analysis of 61,389 in- and outpatients. Abdom Imaging 39:1009-1013
28. Nese N, Martignoni G, Fletcher CD et al (2011) Pure epithelioid PEComas (so-called epithelioid angiomyolipoma) of the kidney: A clinicopathologic study of 41 cases: detailed assessment of morphology and risk stratification. Am J Surg Pathol 35:161-176

29. Fernández-Pello $S$, Hora M, Kuusk T et al (2020) Management of sporadic renal angiomyolipomas: a systematic review of available evidence to guide recommendations from the European Association of Urology Renal Cell Carcinoma guidelines panel. Eur Urol Oncol 3:57-72

30. Bissler JJ, Kingswood JC, Radzikowska E et al (2013) Everolimus for angiomyolipoma associated with tuberous sclerosis complex or sporadic lymphangioleiomyomatosis (EXIST-2): a multicentre, randomised, double-blind, placebo-controlled trial. Lancet 381:817-824

31. Ishigami K, Jones AR, Dahmoush L et al (2015) Imaging spectrum of renal oncocytomas: a pictorial review with pathologic correlation. Insights Imaging 6:53-64

32. Adamy A, Lowrance WT, Yee DS et al (2011) Renal oncocytosis: management and clinical outcomes. JUrol 185:795-801

Hinweis des Verlags. Der Verlag bleibt in Hinblick auf geografische Zuordnungen und Gebietsbezeichnungen in veröffentlichten Karten und Institutsadressen neutral. 